\title{
Review Article \\ The Function of miRNA in Hepatic Cancer Stem Cell
}

\author{
Wei Qi, ${ }^{1}$ Weicheng Liang, ${ }^{2,3}$ Huiqing Jiang, ${ }^{1}$ and Mary Miuyee Waye ${ }^{2,3}$ \\ ${ }^{1}$ Department of Gastroenterology, The Second Hospital of Hebei Medical University, Hebei Key Laboratory of Gastroenterology, \\ Hebei Institute of Gastroenterology, Shijiazhuang, Hebei 23000, China \\ ${ }^{2}$ School of Biomedical Sciences, The Chinese University of Hong Kong, Shatin, Hong Kong \\ ${ }^{3}$ Croucher Laboratory for Human Genomics, The Chinese University of Hong Kong, Shatin, Hong Kong \\ Correspondence should be addressed to Huiqing Jiang; huiqingjiang@yahoo.com.cn \\ and Mary Miuyee Waye; mary-waye@cuhk.edu.hk
}

Received 3 June 2013; Revised 27 October 2013; Accepted 8 November 2013

Academic Editor: Manoor Prakash Hande

Copyright (c) 2013 Wei Qi et al. This is an open access article distributed under the Creative Commons Attribution License, which permits unrestricted use, distribution, and reproduction in any medium, provided the original work is properly cited.

\begin{abstract}
Hepatocellular carcinoma (HCC) is the fifth most common cancer worldwide and ranks third in the leading causes of cancer patient's death. Cancer stem cells (HSCs), also known as tumor-initiating cells, have been reported in multiple subtypes of HCC and are considered as the master regulators of HCC initiation, chemotherapy drug resistance, tumor metastasis, and progression. In spite of their clinical importance, the detailed mechanism about how HSCs are intricately regulated in the molecular level remains elusive. MicroRNA (miRNA), a class of newly emerging small noncoding RNAs, has been demonstrated to serve as a vital player in modulating a number of biological activities ranging from embryogenesis to programmed cell death as well as the maintenance of HSCs. In this review, we synthesize these latest findings of miRNA regulation of HSCs and try to elucidate their mechanistic roles in orchestrating cellular equilibrium. This recent progress underlies the functional role of miRNA in cellular transformation of liver cancer, which has largely extended our knowledge how HSCs are controlled by miRNA network, and in the development of novel miRNA-based anticancer therapies specifically targeting HSCs in the coming future.
\end{abstract}

\section{Introduction}

Twenty years ago, two small noncoding but functional RNAs, lin-4 and let-7, were first identified to control the developmental timing in the nematode Caenorhabditis elegans $[1,2]$. Since then, extensive studies have been conducted to uncover the functional role of miRNA in multiple biological activities, ranging from embryonic development to cell death [3-7]. Moreover, compelling evidence has revealed that miRNA, the small endogenous noncoding RNA, serves as an important player in modulating diverse cellular process through targeting the protein-coding genes or even long noncoding RNAs. Hence, the discovery of microRNA (miRNA) largely extends our understanding about gene expression and regulation. It is estimated that the human genome may encode more than 1000 miRNAs and a number of miRNAs are highly conserved across a wide range of distinct species $[8,9]$.

\section{The Biogenesis of miRNA}

The biogenesis of miRNAs includes multiple steps including transcription, nuclear processing, exportation, and cytoplasmic processing as well as translation inhibition or activation $[10,11]$. miRNAs are transcribed as primary miRNAs (primiRNAs) by RNA polymerase II or III with $5^{\prime}$ end caps and $3^{\prime}$ end poly-A tails [12-14]. The primary miRNAs from intergenic region are processed by the protein complex including nuclease, DiGeorge syndrome critical region gene 8 (DGCR8) and Drosha, whereas the other primary miRNAs from intragenic region are processed by spliceosomes [1517]. After modifications from the above microprocessors, the precursor miRNAs (pre-miRNAs) derived from the primary miRNAs are exported to the cytoplasm with the assistance of the protein Exportin-5 [18]. In the cytoplasm, the pre-miRNAs are cleaved and processed by the nuclease termed Dicer [13] and its partner, human immunodeficiency 


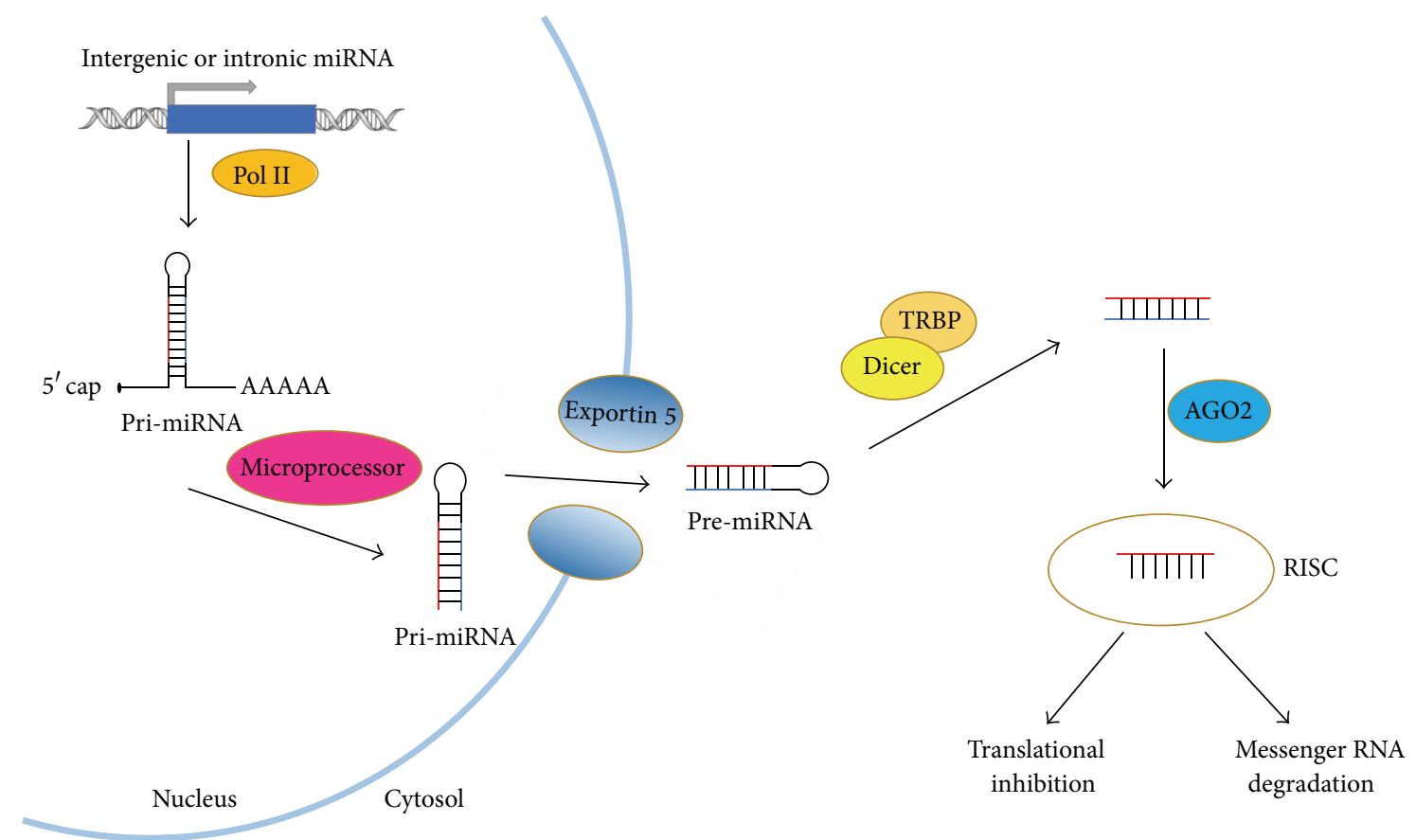

FIGURE 1: Schematic overview of canonical miRNA biogenesis. The pri-miRNA is transcribed from intergenic or intronic region and subsequently spliced by microprocessors, namely, Drosha/DGCR8 or spliceosome. On export from the nucleus to cytosol via Exportin 5, the pre-miRNA is incorporated into RISC complex after Dicer cleavage. Being unwound in RISC complex, one strand stays in the RISC complex as the mature miRNA while the other strand undergoes degradation. Binding to target messenger RNA by miRNAs in RISC complex is followed by translation inhibition and/or mRNA degradation in the cytoplasm.

virus transactivating response RNA binding protein (TRBP), which produces final products around 21-23 nucleotides in length with $5^{\prime}$ phosphates and 2-nucleotide $3^{\prime}$ overhangs. Finally, two complementary small RNA fragments are generated and subsequently designated as $-5 \mathrm{p}$ and $-3 \mathrm{p}$. The mature miRNAs are incorporated into the RNA-induced silencing complex (RISC) and then mediate messenger RNA degradation and/or translational inhibition (Figure 1) [10, 11].

\section{RNA Activation}

Intriguingly, emerging evidence indicates that small RNAs also act as transcriptional activators via interacting with promoter region or untranslated region [19-21]. Vasudevan et al. found that miRNAs inhibit gene translation in proliferating state but stimulate it in a quiescent state. They further demonstrated that the AGO2-miR369-3 complex serves as scaffold proteins to recruit the fragile X-related protein 1 (FXR1) and subsequently activates mRNA translation by binding to the $3^{\prime}$ UTR region of TNF $\alpha$ mRNA [19]. Ørom et al. unraveled that miR-10a interacts with the $5^{\prime}$ UTR region of multiple ribosomal protein mRNAs and plays an important role in translational induction of these mRNAs to fight against survival stress including amino acid starvation [20]. Regardless of the untranslated region, some groups also identified several small RNAs stimulating gene translation through interacting with specific promoter region [22-25]. For instance, Li et al. identified that several artificially synthesized dsRNAs or endogenous miRNA could activate gene transcription of ECadherin by binding to the promoter region of E-Cadherin $[23,24]$. Nevertheless, the detailed mechanism underlying RNA activation remains largely unknown at the present time. Further investigations are badly needed to elucidate the molecular mechanism regarding how miRNAs activate gene expression via interacting with promoter region or untranslated region.

\section{Regulation of Embryonic Stem Cells by miRNA}

Recent studies highlight the function of miRNAs in controlling the self-renewal and pluripotency as well as differentiation of progeny cells. The overall function of miRNAs in mouse embryonic stem cells (ESCs) has been studied by generating the Dicer-null mice. The ESCs derived from Dicernull mice display embryonic lethality as well as severe defects in differentiation both in vitro and in vivo, indicating that Dicer participates in diverse essential biological activities ranging from embryogenesis to maintaining the genome stability [26, 27]. Moreover, researchers also generated the DGCR8-deficient mouse ESCs and these mice show defects in cell proliferation and cell-cycle transition. Further experiments verified that DGCR8 is fundamental for miRNA biogenesis and that miRNAs are master regulator of embryonic cell differentiation [28].

Since Shinya Yamanaka's generating the induced pluripotent stem cells (iPS cells) first from mouse fibroblast and 
subsequently from human via using pluripotent stem cell transcription factor including OCT3/4, SOX2, KLF4, and MYC, plenty of methods including viral transduction or small-molecule compounds for producing iPS cells in vitro have been developed [29-32]. These new approaches are frequently used in combination with small molecules that serve as potent enhancers of iPS cell development [31, 32]. Currently, some miRNA clusters, highly expressed in embryonic stem cells, were identified to promote iPS cells reprogramming in conjunction with the Yamanaka factors (OCT3/4, SOX2, KLF4, and MYC) [33, 34]. Nevertheless, how these miRNAs promote iPS cells reprogramming remains elusive but may be partially related to their ability to orchestrate cell cycle transition and cell death [33]. Of the miRNAs preferentially expressed in iPS cells, the miR302/367 cluster is directly regulated by transcription factors SOX2 and OCT3/4, both of which are essential for iPS reprogramming [35]. In addition, some transcription factors involved in maintaining stem cell pluripotency, including SOX2, OCT3/4, NANOG, and TCF3, were also found to directly bind to the promoter regions of ESC-specific miRNAs [36]. On the other hand, some ESC-specific miRNAs were found to directly target the pluripotency genes at the translational level. For instance, miR-134, miR-296, and miR-470 are significantly upregulated during the differentiation of mouse embryonic stem cells after induction with retinoic-acid and target NANOG, OCT3/4, and SOX2 by binding to their coding regions, inducing the mouse embryonic stem cells morphology changes and resulting in a novel phenotype [37]. The RNA binding protein lin-28, a biomarker of undifferentiated ESCs, is a bona fide target for let-7 during developmental commitment [38, 39]. Conversely, further studies illustrated that the biogenesis of let-7 family is tightly modulated by lin-28. For instance, lin28 could suppress let- 7 maturation by binding to the loop of the primary let-7 $[38,40]$ or the stem part of precursor let$7[39,41]$. Thus, let-7 and lin-28 form an automatic negative feedback loop to precisely modulate each other's expression level.

\section{Hepatic Cancer Stem Cells and miRNA}

Hepatocellular carcinoma (HCC), affecting more than half million individuals annually, is the fifth leading cause of cancer and ranks third in cancer mortality worldwide [42]. The majority of HCC patients are diagnosed in advanced stages with ineffective therapeutic options and unfavorable prognosis [43]. Resection and transplantation are not effective for HCC in advanced stages [44]. Furthermore, the emergence of cellular resistance to current chemotherapy and radiotherapy modalities leaves this disease with frequent occurrence of relapses $[45,46]$. In order to identify novel treatment strategies and tumor markers associated with tumorigenesis of HCC, intensive efforts have been made [47].

A growing body of evidence in cancer biology has indicated that tumor tissues are organized in a hierarchy consisted of heterogeneous cell populations, and the capability to maintain tumorigenesis exclusively relies on a small population of cells called cancer stem cells (CSCs) [48]. Cancer stem cells are key players sustaining tumor formation and growth and are also endowed with stem cell properties $[49,50]$, including the self-renewal ability and pluripotency. Current chemotherapy and radiotherapy modalities eliminate the bulk of cancerous cells but fail to eliminate all of CSCs that maintain a high capacity for renewal $[46,51]$. Similar to normal stem cells, cancer stem cells generate their progeny and have the ability to reconstitute tumors. Currently, it is reported that CSCs exist in glioblastoma, leukemia, prostate, breast, lung, gastric, and colon cancer [49, 52-57]. Similar to many other cancers, CSCs in HCC have also been proven [58].

It is postulated that CSCs derive from stem cells carrying mutations or dedifferentiated mature cells. It is believed that transformation from a normal cell to a cancerous cell requires approximately 3 to 6 genetic events [59]. As stem cells maintain longer life span compared to their progeny, it is postulated that stem cells have the highest potential to accumulate the requisite number of mutations to disturb intrinsic mechanisms regulating normal cell metabolisms and proliferation. On the other side, the dedifferentiation of mature cells possibly happens in tumorigenesis. For example, in the process of epithelial mesenchymal transition (EMT), mature cell becomes more "stem cell-like" with certain upregulated transcription factors, such as SOX2 and KLF4.

Based on specific surface markers and functional properties, hepatic CSCs have been isolated from heterogeneous tumor tissues. Various markers have been found for hepatic cancer stem cells, including CD133, CD90, and EpCAM. Depending on the surrogate characteristics, functional assays to isolate hepatic CSCs have been developed, including side population approach, ALDEFLUOR-approach, sphere formation, and asymmetric division $[60,61]$. Due to the plasticity of CSCs, it is not appropriate to identify and isolate hepatic CSCs by a single marker or functional assay. It is necessary to define hepatic CSCs by an integrated approach combining proper immunogenic markers and functional assay.

Several signaling pathways have been unraveled in hepatocarcinogenesis including MET, MYC, TGF- $\beta$, Hedgehog, p53, WNT/ $\beta$-Catenin, and EGF. Many of them overlap with pathways associated with hepatic progenitor cells. The functions of miRNA and other noncoding RNAs in hepatic CSCs have also been reported.

Based on the computational and experimental evidence, it has been estimated that miRNAs encoded by the human genome could modulate around $60 \%$ of mammalian genes, highlighting the importance of miRNAs in orchestrating gene expression. Furthermore, it has been reported that a single miRNA may affect the expression levels of a number of target genes $[62,63]$. Together, it will not be surprising that even a single type of miRNA may enable normal cells to be transformed into cancerous cells [64-68]. Two recent studies highlight the functional role of miRNAs in regulating carcinogenesis through modulating the stemness properties of CSCs [69, 70] (Figure 2).

By detecting EpCAM and Alpha-fetoprotein (AFP) expression status in conjunction with transcriptome analysis in HCC tumor specimens, Yamashita et al. distinguished two HCC subtypes for prognostic prediction, and they are $\mathrm{EpCAM}^{+} \mathrm{AFP}^{+} \mathrm{HCC}$ (referred to as hepatic stem cell-like 


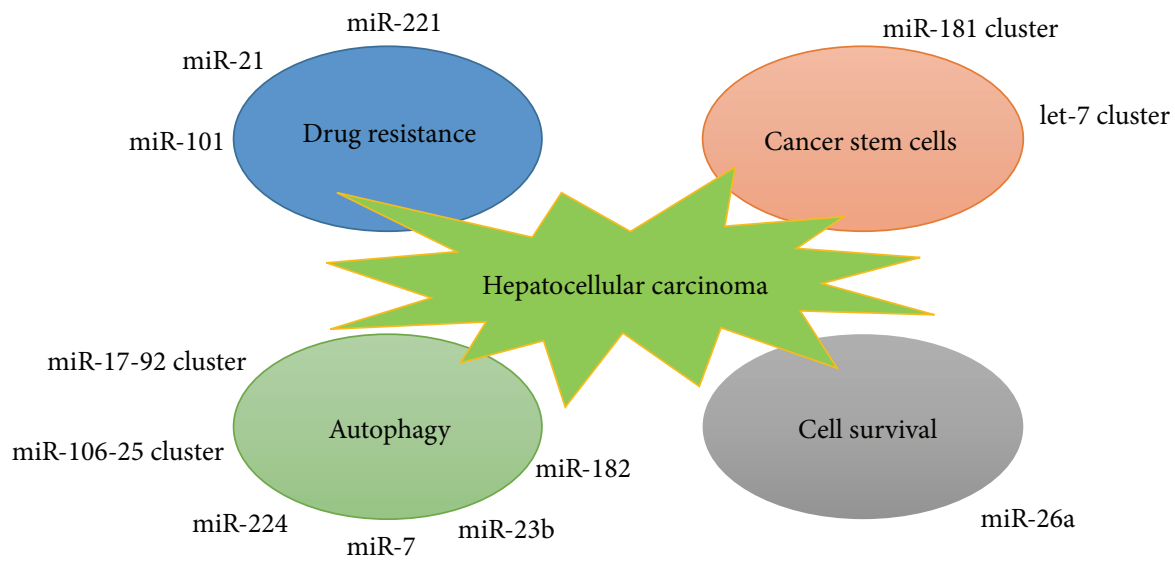

FIGURE 2: The role of miRNAs in regulating key properties of hepatic cancer cells. Several miRNAs, via targeting a variety of downstream signaling pathways, act synergistically to regulate several key biological properties of liver cancer cells including drug resistance, stemness properties, cell survival, and autophagy.

HCC; HpSC-HCC) with enhanced metastatic properties and poor outcome and $\mathrm{EpCAM}^{-} \mathrm{AFP}^{-} \mathrm{HCC}$ (referred to as mature hepatocyte-like HCC; $\mathrm{MH}-\mathrm{HCC}$ ) with good prognosis [71, 72]. Later, by comparing miRNA expression profiles between hepatic stem cell-like hepatocellular carcinoma (HpSC-HCC) and mature hepatocyte-like hepatocellular carcinoma (MH-HCC), Ji et al. unraveled the specific miRNAs preferentially expressed in HpSC-HCC and demonstrated that the highly conserved miR-181 family are highly expressed in $\mathrm{EpCAM}^{+} \mathrm{AFP}^{+}$cells isolated from $\mathrm{AFP}^{+} \mathrm{HCC}$ tumor specimens [69]. Further experiments elucidated that miR-181 family exert their function through targeting Caudal type homeobox transcription factor 2 (CDX2), GATA binding protein 6 (GATA6), and Nemo-like kinase (NLK), which are essential for hepatic cell differentiation and Wnt pathway [69].

After isolating HSCs from liver cancer tissues by using positive markers including OCT3/4, CD133, Nestin, and AFP as well as Carcino-embryonic antigen (CEA), Meng et al. evaluated the miRNA signatures in human HSCs by microarray-based approach followed by the validation with real-time PCR [70]. Among the miRNAs upregulated in HSCs, let-7 and miR-181 family are of great interest because of their vital role in regulating stemness characteristics (Figure 2). They also confirmed that these two miRNA families are regulated by IL- 6 and Twist, respectively.

The sophisticated molecular alternation which occurs during hepatocarcinogenesis provides a long-lasting challenge for researchers. To address this issue, numerous investigations have been conducted. However, although encouraging progress elucidating molecular mechanism on miRNA and HSCs has been achieved, there is a substantial need for more thorough investigations on how miRNAs are involved in HSCs development and tumor progression.

\section{6. miRNA as a Therapeutic Tool in HCC: From Bench to Bedside}

Currently, the discovery of aberrant miRNA expression profiles in liver cancer largely extends our understanding of
HCC and recent studies have shown that miRNA emerges as a promising tool with higher specificity and accuracy for clinical diagnosis and prognosis [73].

Compelling evidences have implied the potential application of miRNA as a novel strategy in cancer therapy for HCC. At present, to develop the potential therapeutic tools for caner therapy, researchers utilize viral vector system to elevate tumor suppressive miRNAs that eliminate carcinogenesis or anti-miRNA oligonucleotides (to repress the oncogenic miRNAs) which promotes tumorigenesis [11].

Among the established approaches for the in vivo miRNA delivery, the adenoassociated viral vectors were found to be a promising therapeutic strategy for cancer therapy because of the lower risk of vector-related toxicities as well as higher gene transfer efficacy $[74,75]$. Kota et al. found out that the expression level of miR-26a is significantly downregulated in the MYC-induced hepatocarcinoma murine model (tet-oMYC; LAP-tTA mice), and this result was further confirmed by detecting the expression profiling of miR-26a in human HCC and normal liver biopsies [75]. Subsequent experiments showed that the tumor suppressive role of miR-26a may be related to their ability to regulate the cell cycle progression via targeting Cyclin D2 and Cyclin E2, two influential players in G1/S cell phase transition. To further characterize the function of miR-26a in vivo and in vitro, they utilized an adenoassociated virus carrying miRNA-26a gene to infect HepG2 cells and mouse model with MYC-induced hepatocarcinoma and found out that ectopic expression of miR26a results in blockage of cell proliferation and induction of tumor cell apoptosis, suggesting that delivery of miRNAs with tumor suppressive function may provide a novel strategy to develop miRNA therapy.

Besides viral vectors, artificially synthesized miRNA or anti-miRNA oligonucleotides were also shown to serve as an important therapeutic strategy for cancer therapy. Park et al. evaluated the therapeutic efficacy of anti-miR-221 oligonucleotides with different chemical modification forms [76]. Of 9 modification forms evaluated, a cholesterol-modified isoform of anti-miR-221 oligonucleotides significantly impairs 
in vitro and in vivo cancer cell proliferation, indicating that this target agent may benefit anticancer treatments for HCC patients [76]. In terms of the important biological implication of miR-221 in HCC, Callegari et al. generated a transgenic mouse model with inappropriate overexpression of miR-221 in the liver, which spontaneously develops liver tumors in a number of mice [77]. Further experiments showed that in vivo delivery of antisense $2^{\prime}$ - $O$-methyloligoribonucleotide targeting miR-221 results in a prolonged survival and significant reduction of the number of tumor nodules in murine HCC models [77]. Similarly, restoration of some miRNAs which serve as tumor suppressors could significantly block tumorigenesis and metastasis in vivo [78-80]. It is well characterized that Osteopontin (OPN) is overexpressed in liver cancer patients with enhanced metastasis and poor prognosis, and repression of OPN using neutralizing antibody could significantly weaken cell migration and invasiveness in vitro [81]. The artificial miRNAs, designated for targeting OPN, could significantly inhibit OPN expression in HCCLM3 cell line and result in decreased in vivo tumor growth and lung metastasis through the repression of matrix metalloproteinase 2 (MMP2) and NF- $\kappa$ B pathways [82].

Furthermore, miRNAs are shown to reduce drug sensitivity of tumor cells to chemotherapy. Hepatoma cells with elevated expression of miR-21 were found to lower drug sensitivity to the cellular cytotoxicity induced by Interferon- $\alpha$ (IFN- $\alpha$ ) in conjunction with 5-Fluorouracil (5-FU), and high level of miR-21 in clinical human hepatoma biopsies indicates unfavorable response to the IFN- $\alpha / 5-\mathrm{FU}$ combination chemotherapy [83]. Furthermore, ectopic expression of antimiR-21 oligonucleotides renders HCC cells sensitive to IFN$\alpha / 5-\mathrm{FU}$, and similar results can be achieved by transfection of siRNAs against phosphatase and tensin homologue (PTEN) or programmed cell death 4 (PDCD4), two bona fide targets for miRN-21 [83]. MiR-101, a frequently downregulated miRNA in HCC, sensitizes HepG2 cells to apoptosis induced by serum starvation or chemotherapeutic drugs through targeting myeloid cell leukemia sequence 1 (Mcl-1), a wellcharacterized antiapoptotic member of Bcl-2 family [84].

All together, these novel findings suggest that patients may benefit from specific miRNA adjuvant administration based on diverse miRNA expression profiles in different individuals suffering from HCC.

\section{Role of Autophagy and miRNA in Liver Cancer}

Autophagy is a tightly regulated cellular catabolic process involving the clearance of organelles and macromolecules. This genetically programmed process includes serial steps ranging from initiation of the phagophore assembly site to the formation of double membrane vesicle complex termed autophagosome, which encapsulates cytoplasmic organelles and proteins and subsequently fuses with lysosomes for the degradation of these intracellular constituents [85]. As a constantly changing process, autophagy provides an alternative energy source for the adaptation of immediate or prolonged metabolic stress.
Recent studies identified a number of miRNAs as a novel player in tightly controlling autophagy and maintaining cell viability as well as intracellular homeostasis $[86,87]$. The polycistron miR-17 92 and its paralog miR-106b-25, two miRNA clusters frequently highly expressed in HCC $[88,89]$, were identified as regulators of autophagy by targeting p62, a multifunctional signal transducer modulating transportation of polyubiquitinated proteins for proteasome degradation [90]. Ectopic expression of these two clusters significantly promotes cell proliferation and differentiation capacity of myeloid progenitors. Moreover, several HCC-relevant miRNAs such as miR-182, miR-23b, miR-101, and miR-224 were shown to participate into the regulation of autophagy. Peng et al. demonstrated that miR-182 is significantly upregulated in prostate cancer cells in response to Atorvastatin, a putative inducer of autophagy in prostate cancer cells. Further experiments indicate that the proautophagic capacity of miR182 is partially mediated by its downstream targets Bcl-2 and p21 [91]. By using radioresistant pancreatic cancer cell model, Wang et al. unveiled that overexpression of miR$23 \mathrm{~b}$ attenuates radiation-mediated autophagy and sensitizes cancer cells to irradiation therapy through targeting Atg12 [92]. Tazawa et al. developed an engineered telomerasespecific oncolytic adenovirus with overexpression of tumor suppressive miR-7 and revealed that ectopic expression of miR-7 inhibits cell proliferation and triggers autophagy through a novel E2F1-miR-7-EGFR axis [93]. Very recently, Lan et al. uncovered a noncanonical pathway including miR224 and autophagy in hepatitis B virus (HBV) related HCC patients [94]. Interestingly, mature miR-224 is preferentially localized into the autophagosome, where miR-224 undergoes degradation. However, in the HBV-associated HCC patients, due to unknown mechanism, low level of autophagy occurred and may partially account for the elevated expression of miR-224 in liver cancer [94]. Considering the important role of autophagy in cancer biology, attempts have been made to suppress tumor growth by miRNA-mediated inhibition of autophagy $[95,96]$. However, further investigations are needed to establish the missing link for miRNAs and autophagy.

\section{Conclusion and Perspectives}

Currently, it has been well established that deregulation of miRNA expression significantly contributes to liver cancer progression. In most cases, miRNAs exert their function through induction translational inhibition and target mRNA degradation. Nevertheless, growing evidences suggest that they may possess other yet unknown functions such as behaving as transcription activator in gene regulation. Despite the fact that inspiring progress has been achieved in miRNAmediated gene activation, many questions remain to be further elucidated. On the other hand, the identification of miRNA signatures aberrantly expressed in HCC paves the way to have a better understanding of the classification and assessment for diverse HCC subtypes in cancer patients. However, to achieve this goal, the specificity and accuracy of HCC-associated miRNA signatures need to be further verified with higher efficacy in the future studies. 
The studies reviewed herein aim to highlight the overwhelming evidences regarding miRNA regulation of stemness characteristics of hepatic cancer stem cells, by modulating tumor-suppressive and oncogenic signaling pathways. The aforementioned new findings largely extend our understanding of HSCs regulation and shed light on developing novel therapeutic strategies to fight against chemotherapyresistant HCC tumors. Given that the biogenesis of HSCs involved multiple steps including tumor initiation, epithelial mesenchymal transition, metastasis, and drug resistance to chemotherapy, miRNA-based therapy strategy that specifically attacks HSCs may launch novel firepower to the war against HCC. As individual miRNAs seem to distinctively modulate different aspect of stemness properties of HSCs (Figure 2), complete elimination of cancer stem cells in HCC tumors and the residual cancer cells may require the utility of multiple miRNAs in developing anti-HCC treatments.

\section{Conflict of Interests}

All authors declare that they have no potential conflict of interests.

\section{Acknowledgments}

This work was partially supported by Research Grants to Dr. Huiqing Jiang from the National Natural Science Foundation (China, no. 81170411) and the Natural Science Foundation of Hebei Province (China, no. C2010000530).

\section{References}

[1] R. C. Lee, R. L. Feinbaum, and V. Ambros, "The C. elegans heterochronic gene lin-4 encodes small RNAs with antisense complementarity to lin-14," Cell, vol. 75, no. 5, pp. 843-854, 1993.

[2] B. J. Reinhart, F. J. Slack, M. Basson et al., “The 21-nucleotide let-7 RNA regulates developmental timing in Caenorhabditis elegans," Nature, vol. 403, no. 6772, pp. 901-906, 2000.

[3] A. Rosa and A. H. Brivanlou, "MicroRNAs in early vertebrate development," Cell Cycle, vol. 8, no. 21, pp. 3513-3520, 2009.

[4] M. R. Fabian and N. Sonenberg, "The mechanics of miRNAmediated gene silencing: a look under the hood of miRISC," Nature Structural \& Molecular Biology, vol. 19, no. 6, pp. 586593, 2012.

[5] A. Pauli, J. L. Rinn, and A. F. Schier, "Non-coding RNAs as regulators of embryogenesis," Nature Reviews Genetics, vol. 12, no. 2, pp. 136-149, 2011.

[6] J. L. Rinn and H. Y. Chang, "Genome regulation by long noncoding RNAs," Annual Review of Biochemistry, vol. 81, pp. 145-166, 2012.

[7] W. Filipowicz, S. N. Bhattacharyya, and N. Sonenberg, "Mechanisms of post-transcriptional regulation by MicroRNAs: are the answers in sight?" Nature Reviews Genetics, vol. 9, no. 2, pp. 102114, 2008.

[8] E. Berezikov, V. Guryev, J. van de Belt, E. Wienholds, R. H. A. Plasterk, and E. Cuppen, "Phylogenetic shadowing and computational identification of human MicroRNA genes," Cell, vol. 120, no. 1, pp. 21-24, 2005.
[9] I. Bentwich, A. Avniel, Y. Karov et al., "Identification of hundreds of conserved and nonconserved human MicroRNAs," Nature Genetics, vol. 37, no. 7, pp. 766-770, 2005.

[10] D. P. Bartel, "MicroRNAs: genomics, biogenesis, mechanism, and function," Cell, vol. 116, no. 2, pp. 281-297, 2004.

[11] R. Garzon, G. A. Calin, and C. M. Croce, "MicroRNAs in cancer," Annual Review of Medicine, vol. 60, pp. 167-179, 2009.

[12] G. M. Borchert, W. Lanier, and B. L. Davidson, "RNA polymerase III transcribes human MicroRNAs," Nature Structural \& Molecular Biology, vol. 13, no. 12, pp. 1097-1101, 2006.

[13] Y. Lee, M. Kim, J. Han et al., "MicroRNA genes are transcribed by RNA polymerase II," The EMBO Journal, vol. 23, no. 20, pp. 4051-4060, 2004.

[14] X. Cai, C. H. Hagedorn, and B. R. Cullen, "Human MicroRNAs are processed from capped, polyadenylated transcripts that can also function as mRNAs," RNA, vol. 10, no. 12, pp. 1957-1966, 2004.

[15] Y. Lee, C. Ahn, J. Han et al., "The nuclear RNase III Drosha initiates MicroRNA processing," Nature, vol. 425, no. 6956, pp. 415-419, 2003.

[16] J. G. Ruby, C. H. Jan, and D. P. Bartel, "Intronic MicroRNA precursors that bypass Drosha processing," Nature, vol. 448, no. 7149, pp. 83-86, 2007.

[17] E. Berezikov, W.-J. Chung, J. Willis, E. Cuppen, and E. C. Lai, "Mammalian mirtron genes," Molecular Cell, vol. 28, no. 2, pp. 328-336, 2007.

[18] R. Yi, Y. Qin, I. G. Macara, and B. R. Cullen, "Exportin5 mediates the nuclear export of pre-MicroRNAs and short hairpin RNAs," Genes and Development, vol. 17, no. 24, pp. 30113016, 2003

[19] S. Vasudevan, Y. Tong, and J. A. Steitz, "Switching from repression to activation: MicroRNAs can up-regulate translation," Science, vol. 318, no. 5858, pp. 1931-1934, 2007.

[20] U. A. Ørom, F. C. Nielsen, and A. H. Lund, "MicroRNA-10a binds the $5^{\prime}$ UTR of ribosomal protein mRNAs and enhances their translation," Molecular Cell, vol. 30, no. 4, pp. 460-471, 2008.

[21] J. I. Henke, D. Goergen, J. Zheng et al., "MicroRNA-122 stimulates translation of hepatitis C virus RNA," The EMBO Journal, vol. 27, no. 24, pp. 3300-3310, 2008.

[22] B. A. Janowski, S. T. Younger, D. B. Hardy, R. Ram, K. E. Huffman, and D. R. Corey, "Activating gene expression in mammalian cells with promoter-targeted duplex RNAs," Nature Chemical Biology, vol. 3, no. 3, pp. 166-173, 2007.

[23] L.-C. Li, S. T. Okino, H. Zhao et al., "Small dsRNAs induce transcriptional activation in human cells," Proceedings of the National Academy of Sciences of the United States of America, vol. 103, no. 46, pp. 17337-17342, 2006.

[24] R. F. Place, L.-C. Li, D. Pookot, E. J. Noonan, and R. Dahiya, "MicroRNA-373 induces expression of genes with complementary promoter sequences," Proceedings of the National Academy of Sciences of the United States of America, vol. 105, no. 5, pp. 1608-1613, 2008.

[25] V. Huang, R. F. Place, V. Portnoy et al., "Upregulation of cyclin B1 by miRNA and its implications in cancer," Nucleic Acids Research, vol. 40, no. 4, pp. 1695-1707, 2012.

[26] E. Bernstein, S. Y. Kim, M. A. Carmell et al., "Dicer is essential for mouse development," Nature Genetics, vol. 35, no. 3, pp. 215217, 2003.

[27] C. Kanellopoulou, S. A. Muljo, A. L. Kung et al., "Dicer-deficient mouse embryonic stem cells are defective in differentiation and 
centromeric silencing," Genes and Development, vol. 19, no. 4, pp. 489-501, 2005.

[28] Y. Wang, R. Medvid, C. Melton, R. Jaenisch, and R. Blelloch, "DGCR8 is essential for MicroRNA biogenesis and silencing of embryonic stem cell self-renewal," Nature Genetics, vol. 39, no. 3, pp. 380-385, 2007.

[29] K. Takahashi and S. Yamanaka, "Induction of pluripotent stem cells from mouse embryonic and adult fibroblast cultures by defined factors," Cell, vol. 126, no. 4, pp. 663-676, 2006.

[30] K. Takahashi, K. Tanabe, M. Ohnuki et al., "Induction of pluripotent stem cells from adult human fibroblasts by defined factors," Cell, vol. 131, no. 5, pp. 861-872, 2007.

[31] D. Huangfu, R. Maehr, W. Guo et al., "Induction of pluripotent stem cells by defined factors is greatly improved by smallmolecule compounds," Nature Biotechnology, vol. 26, no. 7, pp. 795-797, 2008.

[32] T. Wang, K. Chen, X. Zeng et al., "The histone demethylases Jhdmla/lb enhance somatic cell reprogramming in a vitaminC-dependent manner," Cell Stem Cell, vol. 9, no. 6, pp. 575-587, 2011.

[33] R. L. Judson, J. E. Babiarz, M. Venere, and R. Blelloch, "Embryonic stem cell-specific MicroRNAs promote induced pluripotency," Nature Biotechnology, vol. 27, no. 5, pp. 459-461, 2009.

[34] F. Anokye-Danso, C. M. Trivedi, D. Juhr et al., "Highly efficient miRNA-mediated reprogramming of mouse and human somatic cells to pluripotency," Cell Stem Cell, vol. 8, no. 4, pp. 376-388, 2011.

[35] D. A. G. Card, P. B. Hebbar, L. Li et al., "Oct4/Sox2-regulated miR-302 targets cyclin D1 in human embryonic stem cell," Molecular and Cellular Biology, vol. 28, no. 20, pp. 6426-6438, 2008.

[36] A. Marson, S. S. Levine, M. F. Cole et al., "Connecting MicroRNA genes to the core transcriptional regulatory circuitry of embryonic stem cells," Cell, vol. 134, no. 3, pp. 521-533, 2008.

[37] Y. Tay, J. Zhang, A. M. Thomson, B. Lim, and I. Rigoutsos, "MicroRNAs to Nanog, Oct 4 and Sox 2 coding regions modulate embryonic stem cell differentiation," Nature, vol. 455, no. 7216, pp. 1124-1128, 2008.

[38] S. R. Viswanathan, G. Q. Daley, and R. I. Gregory, "Selective blockade of MicroRNA processing by Lin28," Science, vol. 320, no. 5872, pp. 97-100, 2008.

[39] I. Heo, C. Joo, J. Cho, M. Ha, J. Han, and V. N. Kim, "Lin28 mediates the terminal uridylation of let-7 precursor MicroRNA," Molecular Cell, vol. 32, no. 2, pp. 276-284, 2008.

[40] M. A. Newman, J. M. Thomson, and S. M. Hammond, "Lin28 interaction with the Let-7 precursor loop mediates regulated MicroRNA processing," RNA, vol. 14, no. 8, pp. 1539-1549, 2008.

[41] A. Rybak, H. Fuchs, L. Smirnova et al., "A feedback loop comprising lin-28 and let-7 controls pre-let-7 maturation during neural stem-cell commitment," Nature Cell Biology, vol. 10, no. 8, pp. 987-993, 2008.

[42] T. M. Block, A. S. Mehta, C. J. Fimmel, and R. Jordan, "Molecular viral oncology of hepatocellular carcinoma," Oncogene, vol. 22, no. 33, pp. 5093-5107, 2003.

[43] F. Pons, M. Varela, and J. M. Llovet, "Staging systems in hepatocellular carcinoma," HPB, vol. 7, no. 1, pp. 35-41, 2005.

[44] W. T. Kassahun, J. Fangmann, J. Harms, J. Hauss, and M. Bartels, "Liver resection and transplantation in the management of hepatocellular carcinoma: a review," Experimental and Clinical Transplantation, vol. 4, no. 2, pp. 549-558, 2006.
[45] M. T. Kuo, "Redox regulation of multidrug resistance in cancer chemotherapy: molecular mechanisms and therapeutic opportunities," Antioxidants and Redox Signaling, vol. 11, no. 1, pp. 99133, 2009.

[46] R. Morrison, S. M. Schleicher, Y. Sun et al., "Targeting the mechanisms of resistance to chemotherapy and radiotherapy with the cancer stem cell hypothesis," Journal of Oncology, vol. 2011, Article ID 941876, 13 pages, 2011.

[47] S. A. Gonzalez and E. B. Keeffe, "Diagnosis of hepatocellular carcinoma: role of tumor markers and liver biopsy," Clinics in Liver Disease, vol. 15, no. 2, pp. 297-306, 2011.

[48] S. Ma, K.-W. Chan, L. Hu et al., "Identification and characterization of tumorigenic liver cancer stem/progenitor cells," Gastroenterology, vol. 132, no. 7, pp. 2542-2556, 2007.

[49] W. Chen, X. Zhang, C. Chu et al., "Identification of $\mathrm{CD}^{44+}$ cancer stem cells in human gastric cancer," Hepato-gastroenterology, vol. 60, no. 127, pp. 949-954, 2013.

[50] Y. Wang, Y. Yu, A. Tsuyada et al., "Transforming growth factor$B$ regulates the sphere-initiating stem cell-like feature in breast cancer through miRNA-181 and ATM," Oncogene, vol. 30, no. 12, pp. 1470-1480, 2011.

[51] P. B. Gupta, T. T. Onder, G. Jiang et al., "Identification of selective inhibitors of cancer stem cells by high-throughput screening," Cell, vol. 138, no. 4, pp. 645-659, 2009.

[52] Z. Huang, L. Cheng, O. A. Guryanova, Q. Wu, and S. Bao, "Cancer stem cells in glioblastoma-molecular signaling and therapeutic targeting," Protein and Cell, vol. 1, no. 7, pp. 638655, 2010.

[53] J. C. Y. Wang and J. E. Dick, "Cancer stem cells: lessons from leukemia," Trends in Cell Biology, vol. 15, no. 9, pp. 494-501, 2005.

[54] E. S. Polson, J. L. Lewis, H. Celik et al., "Monoallelic expression of TMPRSS2/ERG in prostate cancer stem cells," Nature Communications, vol. 4, article 1623, 2013.

[55] M. S. Schieber and N. S. Chandel, "ROS links glucose metabolism to breast cancer stem cell and EMT phenotype," Cancer Cell, vol. 23, no. 3, pp. 265-267, 2013.

[56] S. Akunuru, Q. James Zhai, and Y. Zheng, "Non-small cell lung cancer stem/progenitor cells are enriched in multiple distinct phenotypic subpopulations and exhibit plasticity," Cell Death \& Disease, vol. 3, article e352, 2012.

[57] J. Lu, X. Ye, F. Fan et al., "Endothelial cells promote the colorectal cancer stem cell phenotype through a soluble form of Jagged-1," Cancer Cell, vol. 23, no. 2, pp. 171-185, 2013.

[58] S. Ma, T. K. Lee, B.-J. Zheng, K. W. Chan, and X.-Y. Guan, "CD133+ HCC cancer stem cells confer chemoresistance by preferential expression of the Akt/PKB survival pathway," Oncogene, vol. 27, no. 12, pp. 1749-1758, 2008.

[59] W. C. Hahn, C. M. Counter, A. S. Lundberg, R. L. Beijersbergen, M. W. Brooks, and R. A. Weinberg, "Creation of human tumour cells with defined genetic elements," Nature, vol. 400, no. 6743, pp. 464-468, 1999.

[60] T. Chiba, K. Kita, Y.-W. Zheng et al., "Side population purified from hepatocellular carcinoma cells harbors cancer stem celllike properties," Hepatology, vol. 44, no. 1, pp. 240-251, 2006.

[61] S. Muramatsu, S. Tanaka, K. Mogushi et al., "Visualization of stem cell features in human hepatocellular carcinoma reveals in vivo significance of tumor-host interaction and clinical course," Hepatology, vol. 58, no. 1, pp. 218-228, 2013.

[62] D. Baek, J. Villén, C. Shin, F. D. Camargo, S. P. Gygi, and D. P. Bartel, "The impact of MicroRNAs on protein output," Nature, vol. 455, no. 7209, pp. 64-71, 2008. 
[63] M. Selbach, B. Schwanhäusser, N. Thierfelder, Z. Fang, R. Khanin, and N. Rajewsky, "Widespread changes in protein synthesis induced by MicroRNAs," Nature, vol. 455, no. 7209, pp. 58-63, 2008.

[64] L. Gramantieri, M. Ferracin, F. Fornari et al., "Cyclin G1 is a target of miR-122a, a MicroRNA frequently down-regulated in human hepatocellular carcinoma," Cancer Research, vol. 67, no. 13, pp. 6092-6099, 2007.

[65] Y. Wang, A. T. C. Lee, J. Z. I. Ma et al., "Profiling MicroRNA expression in hepatocellular carcinoma reveals MicroRNA-224 up-regulation and apoptosis inhibitor-5 as a MicroRNA-224specific target," Journal of Biological Chemistry, vol. 283, no. 19, pp. 13205-13215, 2008.

[66] Q. W.-L. Wong, R. W.-M. Lung, P. T.-Y. Law et al., "MicroRNA223 Is commonly repressed in hepatocellular carcinoma and potentiates expression of stathmin1," Gastroenterology, vol. 135, no. 1, pp. 257-269, 2008.

[67] A. Budhu, H.-L. Jia, M. Forgues et al., "Identification of metastasis-related MicroRNAs in hepatocellular carcinoma," Hepatology, vol. 47, no. 3, pp. 897-907, 2008.

[68] F. Fornari, L. Gramantieri, M. Ferracin et al., "miR-221 controls CDKN1C/p57 and CDKN1B/p27 expression in human hepatocellular carcinoma," Oncogene, vol. 27, no. 43, pp. 5651-5661, 2008.

[69] J. Ji, T. Yamashita, A. Budhu et al., "Identification of MicroRNA181 by genome-wide screening as a critical player in EpCAMpositive hepatic cancer stem cells," Hepatology, vol. 50, no. 2, pp. 472-480, 2009.

[70] F. Meng, S. S. Glaser, H. Francis et al., "Functional analysis of MicroRNAs in human hepatocellular cancer stem cells," Journal of Cellular and Molecular Medicine, vol. 16, no. 1, pp. 160-173, 2012.

[71] T. Yamashita, M. Forgues, W. Wang et al., "EpCAM and $\alpha$ fetoprotein expression defines novel prognostic subtypes of hepatocellular carcinoma," Cancer Research, vol. 68, no. 5, pp. 1451-1461, 2008.

[72] T. Yamashita, J. Ji, A. Budhu et al., "EpCAM-Positive hepatocellular carcinoma cells are tumor-initiating cells with stem/progenitor cell features," Gastroenterology, vol. 136, no. 3, pp. 1012.e4-1024.e4, 2009.

[73] J. Lu, G. Getz, E. A. Miska et al., "MicroRNA expression profiles classify human cancers," Nature, vol. 435, no. 7043, pp. 834-838, 2005.

[74] M. S. Kumar, S. J. Erkeland, R. E. Pester et al., "Suppression of non-small cell lung tumor development by the let-7 MicroRNA family," Proceedings of the National Academy of Sciences of the United States of America, vol. 105, no. 10, pp. 3903-3908, 2008.

[75] J. Kota, R. R. Chivukula, K. A. O’Donnell et al., “Therapeutic MicroRNA delivery suppresses tumorigenesis in a murine liver cancer model," Cell, vol. 137, no. 6, pp. 1005-1017, 2009.

[76] J.-K. Park, T. Kogure, G. J. Nuovo et al., "miR-221 silencing blocks hepatocellular carcinoma and promotes survival," Cancer Research, vol. 71, no. 24, pp. 7608-7616, 2011.

[77] E. Callegari, B. K. Elamin, F. Giannone et al., "Liver tumorigenicity promoted by MicroRNA-221 in a mouse transgenic model," Hepatology, vol. 56, no. 3, pp. 1025-1033, 2012.

[78] W.-C. Tsai, P. W.-C. Hsu, T.-C. Lai et al., "MicroRNA-122, a tumor suppressor MicroRNA that regulates intrahepatic metastasis of hepatocellular carcinoma," Hepatology, vol. 49, no. 5, pp. 1571-1582, 2009.
[79] Q. Lang and C. Ling, "miR-124 suppresses cell proliferation in hepatocellular carcinoma by targeting PIK3CA," Biochemical and Biophysical Research Communications, vol. 426, no. 2, pp. 247-252, 2012.

[80] X. Zhang, S. Liu, T. Hu, S. Liu, Y. He, and S. Sun, "Upregulated MicroRNA-143 transcribed by nuclear factor kappa B enhances hepatocarcinoma metastasis by repressing fibronectin expression," Hepatology, vol. 50, no. 2, pp. 490-499, 2009.

[81] Q.-H. Ye, L.-X. Qin, M. Forgues et al., "Predicting hepatitis B virus-positive metastatic hepatocellular carcinomas using gene expression profiling and supervised machine learning," Nature Medicine, vol. 9, no. 4, pp. 416-423, 2003.

[82] B.-S. Sun, Q.-Z. Dong, Q.-H. Ye et al., "Lentiviral-mediated miRNA against osteopontin suppresses tumor growth and metastasis of human hepatocellular carcinoma," Hepatology, vol. 48, no. 6, pp. 1834-1842, 2008.

[83] Y. Tomimaru, H. Eguchi, H. Nagano et al., "MicroRNA-21 induces resistance to the anti-tumour effect of interferon- $\alpha / 5$ fluorouracil in hepatocellular carcinoma cells," British Journal of Cancer, vol. 103, no. 10, pp. 1617-1626, 2010.

[84] H. Su, J.-R. Yang, T. Xu et al., "MicroRNA-101, down-regulated in hepatocellular carcinoma, promotes apoptosis and suppresses tumorigenicity," Cancer Research, vol. 69, no. 3, pp. 11351142, 2009.

[85] T. Yorimitsu and D. J. Klionsky, "Autophagy: molecular machinery for self-eating," Cell Death and Differentiation, vol. 12, supplement 2, pp. 1542-1552, 2005.

[86] L. B. Frankel and A. H. Lund, "MicroRNA regulation of autophagy," Carcinogenesis, vol. 33, no. 11, pp. 2018-2025, 2012.

[87] J. Cui, Z. Gong, and H. M. Shen, "The role of autophagy in liver cancer: molecular mechanisms and potential therapeutic targets," Biochimica et Biophysica Acta, vol. 1836, no. 1, pp. 1526, 2013.

[88] F. Yang, Y. Yin, F. Wang et al., "miR-17-5p promotes migration of human hepatocellular carcinoma cells through the p38 mitogen-activated protein kinase-heat shock protein 27 pathway," Hepatology, vol. 51, no. 5, pp. 1614-1623, 2010.

[89] Y. Li, W. Tan, T. W. L. Neo et al., "Role of the miR-106b25 MicroRNA cluster in hepatocellular carcinoma," Cancer Science, vol. 100, no. 7, pp. 1234-1242, 2009.

[90] A. Meenhuis, P. A. van Veelen, H. de Looper et al., "miR17/20/93/106 promote hematopoietic cell expansion by targeting sequestosome 1-regulated pathways in mice," Blood, vol. 118, no. 4, pp. 916-925, 2011.

[91] X. Peng, W. Li, L. Yuan, R. G. Mehta, L. Kopelovich, and D. L. McCormick, "Inhibition of proliferation and induction of autophagy by atorvastatin in PC3 prostate cancer cells correlate with downregulation of $\mathrm{Bcl} 2$ and upregulation of miR-182 and p21," PLoS One, vol. 8, no. 8, Article ID e70442, 2013.

[92] P. Wang, J. Zhang, L. Zhang et al., "MicroRNA 23b regulates autophagy associated with radioresistance of pancreatic cancer cells," Gastroenterology, vol. 145, no. 5, pp. 1133.e12-1143.e12, 2013.

[93] H. Tazawa, S. Yano, R. Yoshida et al., "Genetically engineered oncolytic adenovirus induces autophagic cell death through an E2F1-MicroRNA-7-epidermal growth factor receptor axis," International Journal of Cancer, vol. 131, no. 12, pp. 2939-2950, 2012.

[94] S. H. Lan, S. Y. Wu, R. Zuchini et al., "Autophagy suppresses tumorigenesis of hepatitis B virus-associated hepatocellular carcinoma through degradation of miR-224," Hepatology, 2013. 
[95] Z. Zou, L. Wu, H. Ding et al., "MicroRNA-30a sensitizes tumor cells to cis-platinum via suppressing beclin 1-mediated autophagy," Journal of Biological Chemistry, vol. 287, no. 6, pp. 4148-4156, 2012.

[96] Y. Yu, L. Yang, M. Zhao et al., “Targeting MicroRNA-30amediated autophagy enhances imatinib activity against human chronic myeloid leukemia cells," Leukemia, vol. 26, no. 8, pp. 1752-1760, 2012. 


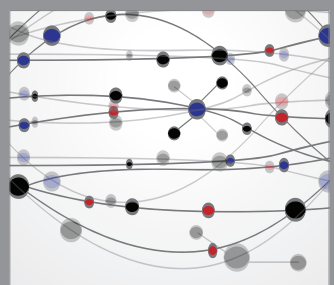

The Scientific World Journal
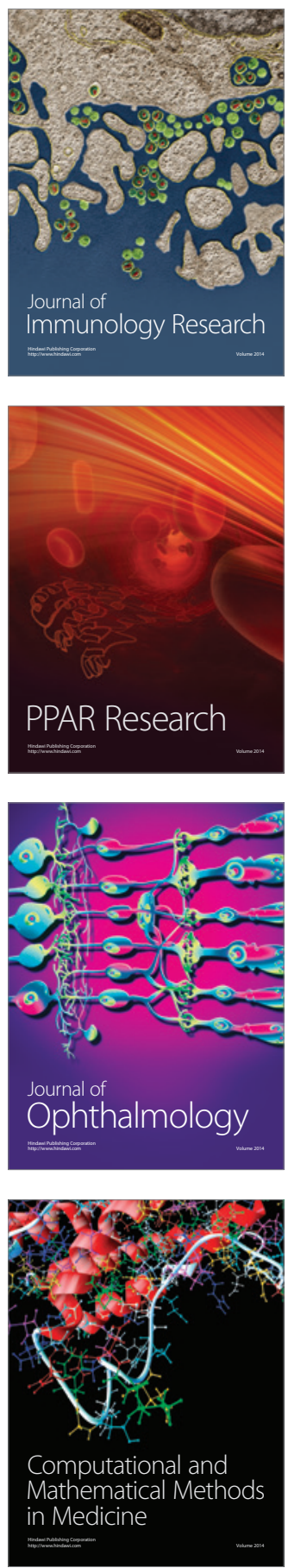

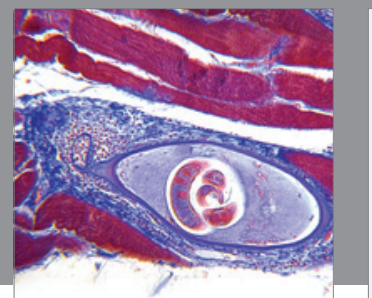

Gastroenterology

Research and Practice
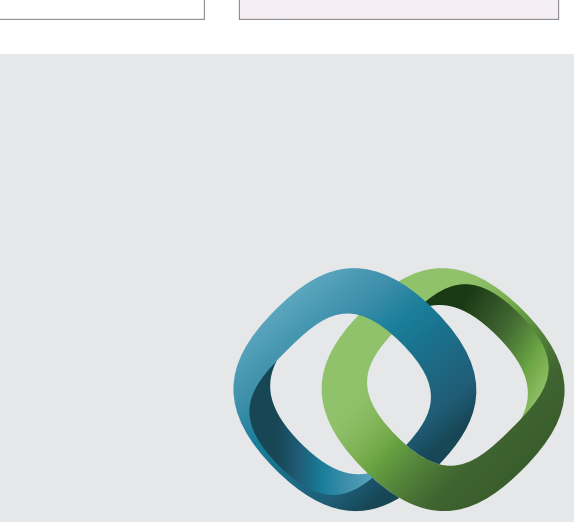

\section{Hindawi}

Submit your manuscripts at

http://www.hindawi.com
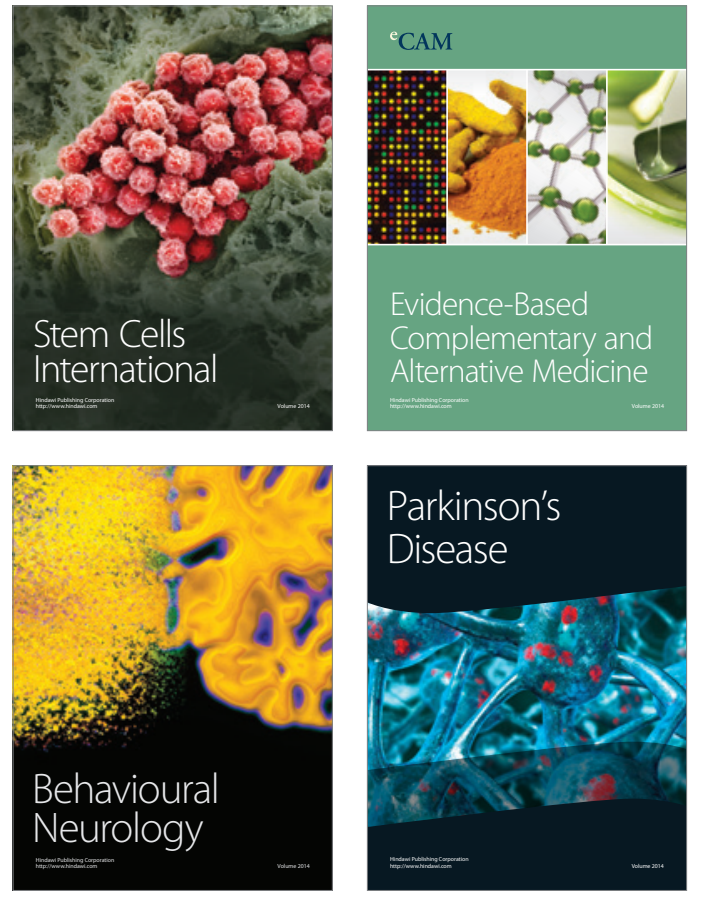
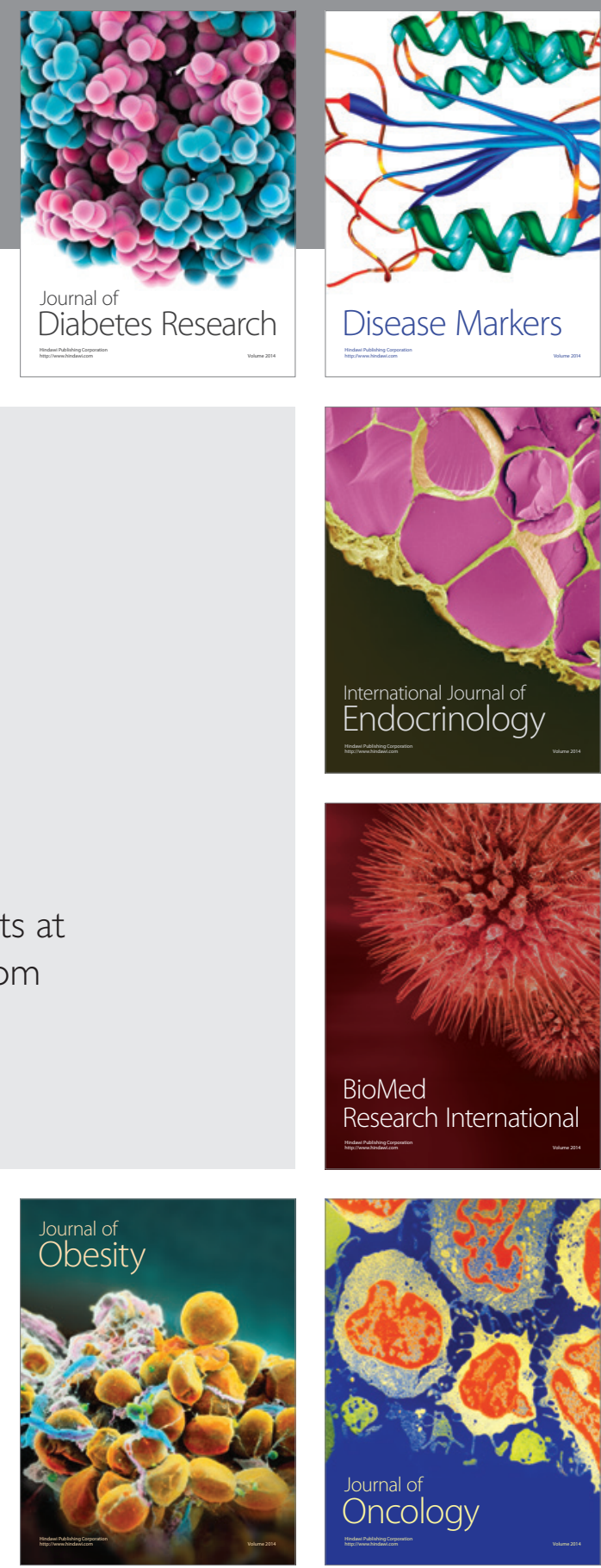

Disease Markers
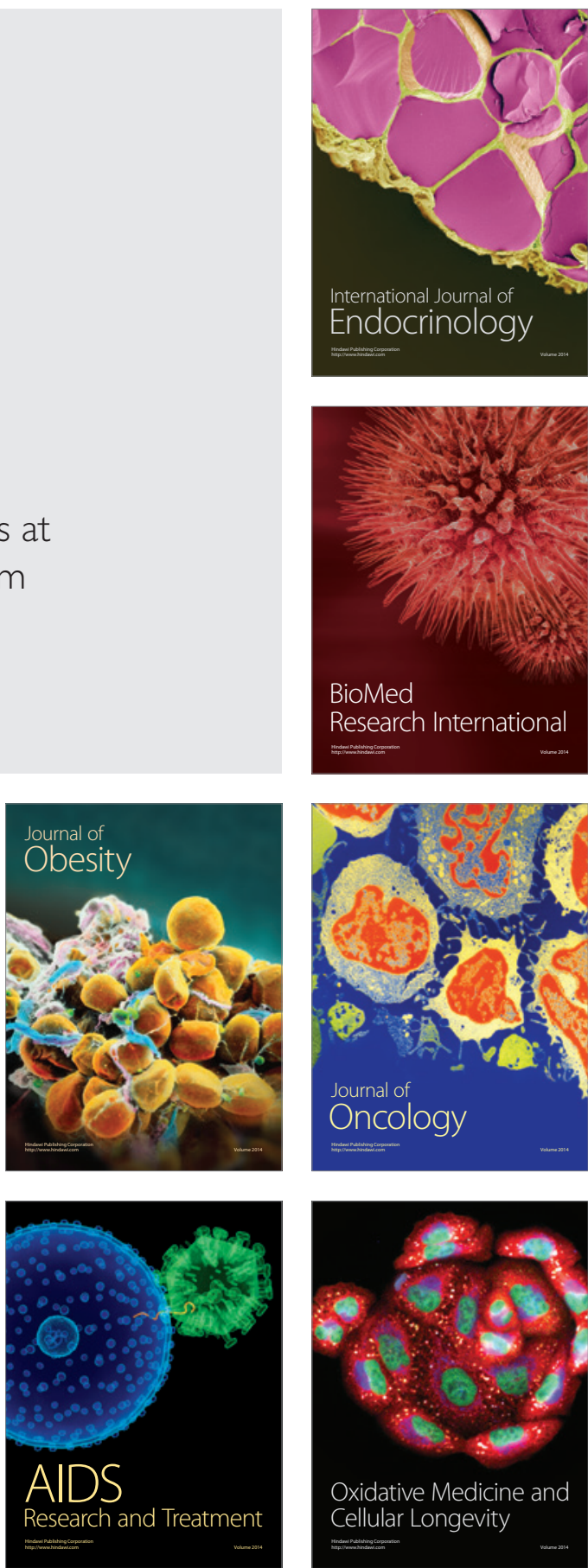